NOTA CIENTÍFICA

\title{
PROPAGAÇÃO POR ESTAQUIA EM PATCHOULI COM DIFERENTES NÚMEROS DE FOLHAS E TIPOS DE ESTACAS
}

\section{CUTTING PROPAGATION OF PATCHOULI WITH DIFFERENT NUMBER OF LEAVES AND TYPES OF CUTTINGS}

\author{
Caroline GARBUIO ${ }^{1}$ \\ Luiz Antonio BIASI ${ }^{2}$ \\ Ana Paula de Jesus KOWALSKI ${ }^{1}$ \\ Diana SIGNOR ${ }^{1}$ \\ Eliana Márcia MACHADO' \\ Cícero DESCHAMPS ${ }^{3}$
}

\begin{abstract}
RESUMO
O patchouli (Pogostemon cablin), pertencente à família Lamiaceae, possui grande interesse comercial pela presença de óleo essencial, contido nas folhas, que é utilizado principalmente na indústria de perfumaria. O objetivo deste trabalho foi avaliar o enraizamento de estacas de patchouli, de acordo com o número de folhas e a região do ramo utilizado para a estaquia. As estacas de patchouli foram obtidas de plantas cultivadas no município de Joinville-SC. Foram instalados dois experimentos com delineamentos experimentais inteiramente casualizados e cinco repetições. No primeiro experimento, foi avaliada a presença de folhas (estacas sem folhas, estacas com uma folha e estacas com duas folhas) usando estacas medianas. No segundo experimento, foi avaliada a posição de coleta das estacas no caule, testando estacas basais, medianas e apicais. As estacas apicais e medianas foram preparadas com um par de folhas e as estacas basais sem folhas. As avaliações foram realizadas aos 30 dias após a estaquia. Estacas apicais e medianas não diferiram entre si, quanto à porcentagem de enraizamento $(93,7 \%$ e $83,7 \%$, respectivamente) e comprimento médio das três maiores raízes por estaca ( $11,1 \mathrm{~cm}$ e 10,2 cm, respectivamente), sendo superiores em relação às estacas basais. O número de raízes emitidas por estaca decresceu significativamente das estacas apicais $(35,5)$ para as basais $(7,3)$. Estacas com duas folhas e uma folha apresentaram maior porcentagem de enraizamento $(71,7 \%$ e $51,7 \%$, respectivamente) e de brotação $(78,4 \%$ e $44,9 \%$, respectivamente) do que as estacas sem folhas. Para o número de raízes emitidas por estaca, aquelas que continham duas folhas $(25,7)$ foram superiores às com uma folha $(15,6)$ e às sem folhas $(2,7)$. Recomenda-se para a propagação por estaquia do patchouli o uso de estacas apicais ou medianas com duas folhas.
\end{abstract}

Palavras-chave: Pogostemon cablin; estaquia; propagação vegetativa.

\begin{abstract}
Patchouli (Pogostemon cablin) belongs to Lamiaceae family and has great comercial interest due the essential oil production in leaves which is used mainly in the perfumary industry. The objective of this work was to evaluate the root formation of patchouli according to leaf and stem cutting positions. The plant material was obtained from Joinville (SC). Two experiments were carried out using a complete randomized design with five replications. The first experiment investigated the effect of leaves on stem cuttings (stems without leaves and with one and two leaves) obtained from medium region of the stems. The second experiment compared the effect of the region of the stem as a source of cuttings and the treatments included basal, medium and apical regions. The stems from apical and medium region had one pair of leaves and the stem from basal region had no leaves. After 30 days the experiment results were evaluated. Stem cuttings collected from the apical and medium regions of the stem showed no statistical difference of rooting percentage $(93,7 \%$ and $83,7 \%$, respectively) and the length of the three great roots of the cuttings $(11,1 \mathrm{~cm}$ and $10,2 \mathrm{~cm}$, respectively), being superior than the basal stem cuttings. The root number on cuttings decreased from apical $(35,5)$ to basal $(7,3)$ region. Stems cuttings with two leaves and with one leaf showed higher root percentage $(71,7 \%$ and $51,7 \%$, respectively) and stem formation $(78,4 \%$ and $44,9 \%$, respectively) than the basal stems without leaves. The root number on stems decreased from stem with two leaves $(25,7)$ than with one leaf $(15,6)$ and withou leaves $(2,7)$. Based on the obtained results, it is recommended the use of stem cuttings from apical and medium regions containing two leaves.
\end{abstract}

Key-words: Pogostemon clablin; cutting; vegetative propagation.

${ }^{1}$ Aluna do Curso de Graduação em Agronomia da UFPR.

2Professor Associado I. Departamento de Fitotecnia e Fitossanitarismo. Setor de Ciências Agrárias. UFPR. Caixa Postal 19.061. CEP 81531-

990. Curitiba-PR. E-mail: biasi@ufpr.br. Bolsista de Produtividade em Pesquisa do CNPq. Autor para correspondência.

${ }^{3}$ Professor Adjunto. Departamento de Fitotecnia e Fitossanitarismo. Setor de Ciências Agrárias. UFPR. 


\section{INTRODUÇÃO}

O patchouli (Pogostemon clablin), pertencente à família Lamiaceae, é nativo das ilhas das Filipinas. As folhas possuem de 5 a $10 \mathrm{~cm}$ de comprimento e 3 a $8,9 \mathrm{~cm}$ de largura. A margem é levemente lobada e os lobos possuem dentes crenato-serrados, os lobos e o ápice da folha são obtusos. Na face inferior da folha existem muitos pelos ao longo das nervuras e ocasionam uma aparência pálida. A variação morfológica do patchouli causada pelas condições de solo, clima e influência do cultivo, dificulta a classificação botânica (GUENTHER, 1972).

O patchouli ( $P$. cablin) prefere ambientes sombreados e com boa distribuição de chuvas. A propagação do patchouli é realizada com estacas caulinares, retiradas das partes jovens da planta. $O$ cultivo se dá por sucessivos cortes da parte aérea, durante o período máximo de quatro anos, sendo necessária a renovação das plantas devido ao baixo rendimento na produção. O seu produto de maior interesse comercial é o óleo essencial, contido nas folhas, utilizado principalmente na indústria de perfumaria (EPAGRI, 2004).

A estaquia é a técnica de propagação vegetativa mais rápida e mais fácil para execução, sendo muito utilizada nas espécies que apresentam maior facilidade para a formação de raízes adventícias. Estudos demonstram que a utilização de diferentes tipos de estacas, com folhas presentes ou ausentes, assim como, a época de coleta influenciam consideravelmente o enraizamento das mesmas (BEZERRA e LEDERMAN, 1995). A presença de folhas e gemas é um dos fatores, que segundo HARTMANN et al. (2002), exerce grande estímulo à iniciação de raízes. Este efeito está relacionado à translocação de carboidratos para a base da estaca, além de auxinas e outros co-fatores importantes para o enraizamento. As auxinas são hormônios vegetais produzidos principalmente nas regiões apicais que, transportados através das células do parênquima para outros locais da planta, participam do seu crescimento e diferenciação (TAIZ e ZEIGER, 2004).

O objetivo deste trabalho foi avaliar o efeito de diferentes números de folhas mantidas nas estacas e de tipos de estacas na propagação de patchouli.

\section{MATERIAL E MÉTODOS}

As estacas de Pogostemon cablin foram obtidas de plantas cultivadas no município de Joinville-SC. Os ramos do crescimento do ano foram coletados e colocados dentro de sacos plásticos, sendo transportados até o Departamento de Fitotecnia e Fitossanitarismo do Setor de Ciências Agrárias da UFPR, em Curitiba-PR.

Foram instalados dois experimentos com delineamento experimental inteiramente casualizado e cinco repetições, no dia 11 de julho de 2006. Num primeiro experimento foi avaliada a posição das estacas no caule, testando estacas basais, medianas e apicais. As estacas apicais e medianas foram preparadas com um par de folhas e as estacas basais sem folhas, pois nessa região do caule não havia folhas. As estacas apicais possuíam consistência herbácea, as medianas consistência semilenhosa e as basais consistência lenhosa. Para esse experimento foram utilizadas 16 estacas por parcela. O segundo experimento foi instalado para avaliar a presença das folhas nas estacas semilenhosas, preparadas com $12 \mathrm{~cm}$ de comprimento a partir da região mediana dos ramos. Os tratamentos foram: estacas sem folhas, estacas com 1 folha e estacas com 2 folhas. Para esse experimento foram utilizadas 12 estacas por parcela.

A estaquia foi realizada em tubetes plásticos com $115 \mathrm{~cm}^{3}$ de capacidade e o substrato utilizado foi o Plantmax HT®. As bandejas contendo as estacas foram mantidas em casa-de-vegetação com sistema de irrigação intermitente, com três turnos de rega, sendo de 15 segundos a cada 15 min das 08:00 às 17:00 h, 15 segundos a cada hora das $17: 00 \mathrm{~h}$ às $23: 00 \mathrm{~h}$ e 15 segundos a cada $3 \mathrm{~h}$ das 23:00 às 08:00 h. Internamente a casa-de-vegetação possuía uma tela Aluminet®.

Após 30 dias foi realizada a avaliação dos experimentos, considerando-se os seguintes parâmetros: número de estacas enraizadas, brotadas, mortas e vivas sem enraizamento por parcela, número e comprimento médio das três maiores raízes por estaca. No experimento com diferente número de folhas também foi avaliada a retenção foliar.

Os dados obtidos foram submetidos à análise de variância e as médias dos tratamentos comparadas pelo teste de Duncan a $5 \%$ de probabilidade.

\section{RESULTADOS E DISCUSSÃO}

No primeiro experimento, não houve diferença significativa entre as posições das estacas no caule para as variáveis brotação e mortalidade. Estacas apicais e medianas não diferiram entre si, quanto a porcentagem de enraizamento, estacas vivas não enraizadas e o comprimento médio das três maiores raízes por estaca, sendo superiores as estacas basais (Tabela 1). Esses resultados são semelhantes aos encontrados por BONA et al. (2005) com duas espécies de carqueja (Baccharis articulata e B. stenocephala), que também apresentaram maior enraizamento e desenvolvimento nas estacas apicais e medianas. EHLERT et al. (2003), ao estudarem a interação do substrato com o tipo de estaca de alfavaca-cravo (Ocimum gratissimum), observaram que houve destaque para as estacas mediana sem folha e apical com folha. NEGRELLE e DONI (2001) também observaram que os ramos semilenhosos com folhas mostraram-se melhores para o preparo das estacas de guaco (Mikania glomerata), pois favorecem a precocidade e estabelecimento. Para a estaquia de fáfia (Pfaffia glomerata), as estacas obtidas das regiões medianas e basais foram melhores do que as aquelas obtidas da região apical, considerando todas as estacas sem folhas (NICOLOSO et al., 1999). Desta forma pode-se observar que a escolha da melhor região do ramo para a estaquia, depende das características específicas de cada planta. 
TABELA 1 - Porcentagem de estacas enraizadas, brotadas, mortas e vivas não enraizadas por parcela, comprimento médio das três maiores raízes e número de raízes emitidas por estaca em função de diferentes tipos de estaca de patchouli. Curitiba, UFPR, 2006.

\begin{tabular}{lccccccc}
\hline $\begin{array}{l}\text { Posição } \\
\text { estaca } \\
\text { caule }\end{array}$ & $\begin{array}{c}\text { da } \\
\text { no }\end{array}$ & $\begin{array}{c}\text { Brotação } \\
(\%)^{1}\end{array}$ & $\begin{array}{c}\text { Mortalidade } \\
(\%)^{1}\end{array}$ & $\begin{array}{c}\text { Enraizamento } \\
(\%)\end{array}$ & $\begin{array}{c}\text { Estacas vivas } \\
\text { não enraizadas } \\
(\%)^{1}\end{array}$ & $\begin{array}{c}\text { Comprimento médio } \\
\text { das três maiores raízes } \\
\text { / estaca }(\mathrm{cm})\end{array}$ & $\begin{array}{c}\text { Número de } \\
\text { raízes / } \\
\text { estaca }\end{array}$ \\
\hline Apical & $57,5 \mathrm{a}$ & $2,5 \mathrm{a}$ & $93,7 \mathrm{a}^{2}$ & $3,7 \mathrm{~b}$ & $11,1 \mathrm{a}$ & $35,5 \mathrm{a}$ \\
Mediana & $48,7 \mathrm{a}$ & $3,7 \mathrm{a}$ & $83,7 \mathrm{a}$ & $12,5 \mathrm{~b}$ & $10,2 \mathrm{a}$ & $28,5 \mathrm{~b}$ \\
Basal & $6,2 \mathrm{a}$ & $0,0 \mathrm{a}$ & $62,5 \mathrm{~b}$ & $37,5 \mathrm{a}$ & $6,5 \mathrm{~b}$ & $7,3 \mathrm{c}$ \\
\hline C.V. $(\%)$ & 80,4 & 71,3 & 14,1 & 35,2 & 16,6 & 21,2 \\
\hline
\end{tabular}

'Dados originais transformados em raiz quadrada $x+1$ para análise.

${ }^{2}$ Médias seguidas pela mesma letra não diferem significativamente pelo teste de Duncan a $5 \%$ de probabilidade.

O número de raízes emitidas por estaca decresceu das estacas apicais para as medianas e para as basais (Tabela 1). Possivelmente a proximidade da gema apical naquele tipo de estaca favoreceu a maior formação de raízes, pois as auxinas produzidas no meristema apical do caule são transportadas de forma basípeta pelas células do parênquima até a base das estacas, onde promovem a indução radicial (TAIZ e ZEIGER, 2004).

A ausência de estacas mortas entre as estacas basais demonstra a maior resistência deste tipo de estaca. Entretanto, a menor porcentagem de enraizamento e o pequeno desenvolvimento do sistema radicial, conferem uma baixa eficiência na estaquia basal e a formação de mudas de qualidade ruim. A presença de muitas estacas vivas não enraizadas pode indicar que para as estacas basais o enraizamento é mais lento, possivelmente pela ausência de folhas. Entretanto, não justifica que seja aguardado o seu possível enraizamento, pois as mudas formadas das estacas apicais e medianas, após 30 dias, apresentavam-se aptas para serem transplantadas, pelo grande desenvolvimento das raízes adventícias e agregação do substrato, evitando danos às raízes quando a muda é retirada do tubete, o que é desejável (MINAMI, 1995).

Estacas com duas folhas e uma folha não diferiram entre si e foram superiores as estacas sem folhas para a porcentagem de enraizamento e brotação. Estacas com ausência de folhas apresentaram maior mortalidade e maior número de estacas vivas não enraizadas, comparando com as anteriores (Tabela 2). A retenção foliar foi superior nas estacas com duas folhas, sendo a porcentagem de enraizamento proporcional à retenção foliar obtida. A permanência das folhas nas estacas é um fator importante para o enraizamento das estacas, conforme já demonstrado em trabalhos com abacateiro (REUVENI e RAVIV, 1981), araçazeiro (NACHTIGAL et al., 1994), goiabeira (PEREIRA et al., 1983) e videira (BIASI et al., 1997).

TABELA 2 - Porcentagem de estacas enraizadas, brotadas, mortas e vivas não enraizadas por parcela, porcentagem de retenção foliar, comprimento médio das três maiores raízes e número de raízes emitidas por estaca, em função do número de folhas mantidas na estaca mediana de patchouli. Curitiba, UFPR, 2006.

\begin{tabular}{|c|c|c|c|c|c|c|c|}
\hline $\begin{array}{lc}\text { Número } & \text { de } \\
\text { folhas } & \text { por } \\
\text { estaca } & \end{array}$ & $\begin{array}{c}\text { Enraíza- } \\
\text { mento } \\
(\%)^{1}\end{array}$ & $\begin{array}{c}\text { Brotação } \\
(\%)^{1}\end{array}$ & $\begin{array}{c}\text { Mortali- } \\
\text { dade }(\%)^{1}\end{array}$ & $\begin{array}{c}\text { Estacas vivas } \\
\text { não } \\
\text { enraizadas } \\
(\%)^{1} \\
\end{array}$ & $\begin{array}{l}\text { Retenção } \\
\text { foliar (\%) }\end{array}$ & $\begin{array}{l}\text { Comprimento } \\
\text { médio das três } \\
\text { maiores raízes / } \\
\text { estaca }(\mathrm{cm})^{1}\end{array}$ & $\begin{array}{c}\text { Número } \\
\text { de raízes / } \\
\text { estaca }^{1}\end{array}$ \\
\hline 2 folhas & $71,7 \mathrm{a}^{2}$ & $78,4 \mathrm{a}$ & $8,3 \mathrm{~b}$ & $20,0 \mathrm{~b}$ & $69,2 \mathrm{a}$ & $8,8 \mathrm{a}$ & $25,7 \mathrm{a}$ \\
\hline 1 folha & $51,7 \mathrm{a}$ & 44,9 a & $16,7 \mathrm{ab}$ & $31,7 \mathrm{ab}$ & $45,0 \mathrm{~b}$ & $8,7 \mathrm{a}$ & $15,6 b$ \\
\hline Sem folha & $25,0 \mathrm{~b}$ & $11,7 \mathrm{~b}$ & $30,0 \mathrm{a}$ & $45,0 \mathrm{a}$ & - & $5,5 \mathrm{a}$ & $2,7 \mathrm{c}$ \\
\hline C.V. (\%) & 19,2 & 29,7 & 39,4 & 27,6 & 16,6 & 15,5 & 17,9 \\
\hline
\end{tabular}

TDados originais transformados em raiz quadrada $x+1$ para análise.

${ }^{2}$ Médias seguidas pela mesma letra não diferem significativamente pelo teste de Duncan a $5 \%$ de probabilidade.

O número de raízes emitidas por estaca decresceu das estacas com duas folhas para as com uma folha e para as sem folha (Tabela 2). Possivelmente as folhas promoveram o estímulo à iniciação de raízes pela translocação de carboidratos para a base da estaca, além da produção de auxinas e outros co-fatores importantes para o enraizamento (HARTMANN et al., 2002). Para diversos portaenxertos de videira o aumento da área foliar promoveu uma emissão de maior número de raízes, proporcionando maior massa e volume de raízes adventícias por estaca (BIASI et al., 1997).

Os resultados desse trabalho com patchouli foram semelhantes ao trabalho com cipómil-homens (Aristolochia triangularis), onde as estacas com folhas inteiras apresentaram maior porcentagem de enraizamento e maior desenvolvimento de raízes com relação às estacas sem folhas (CORRÊA e BIASI, 2003). Para a estaquia de erva-cidreira-brasileira (Lippia alba), BIASI e COSTA (2003) observaram que estacas medianas contendo quatro folhas tiveram maior desenvolvimento radicial comparando com as sem folhas. Na estaquia semilenhosa de guaco (Mikania glomerata) também houve aumento do enraizamento com o preparo de estacas com maior área foliar (LIMA et al., 2003). Na estaquia semilenhosa de louro (Laurus nobilis), melhores respostas de enraizamento foram obtidas com quatro folhas por estaca, em relação ao uso de apenas duas (FOCHESATO et al., 2006). 


\section{CONCLUSÃO}

Recomenda-se para a estaquia de patchouli a utilização de estacas apicais e medianas com duas folhas.

\section{AGRADECIMENTOS}

Os autores agradecem a Secretaria de Estado de Ciência e Tecnologia (SETI) / Governo do Estado do Paraná pelo apoio financeiro e concessão de bolsa para as alunas de graduação.

\section{REFERÊNCIAS}

1. BEZERRA, J.E.F.; LEDERMAN, I.E. Propagação vegetativa por estaquia da aceroleira. In: SÃO JOSÉ, A.R.; ALVES, R.E. Acerola no Brasil, produção e mercado. Vitória da Conquista: UESB, 1995. p. 32-40.

2. BIASI, L.A; COSTA, G. Propagação vegetativa de Lippia alba. Ciência Rural, v. 33, n. 3, p. 455-459. 2003.

3. BIASI, L.A. ; POMMER, C.V. ; PINO, P.A.G.S. Propagação de porta-enxertos de videira mediante estaquia semilenhosa. Bragantia, v. 56, n. 2, p. 367-376, 1997.

4. BONA, C.M. de; BIASI, L.A; ZANETTE, F; NAKASHIMA, T. Estaquia de três espécies de Baccharis. Ciência Rural, v. 35, n. 1, p. 223-226, 2005.

5. CORREA, C.F; BIASI, L.A. Área foliar e tipo de substrato na propagação por estaquia de cipó-mil-homens (Aristolochia triangularis Cham. Et Schl.) Revista Brasileira de Agrociência, v. 9, n. 3, p. 233-235. 2003.

6. EHLERT, P.A.D; LUZ, J.M.Q.; INNECCO, R. Propagação vegetativa de alfavaca-cravo utilizando diferentes tipos de estacas e substratos. Horticultura Brasileira, v. 22, n. 1, p. 10-13. 2003.

7. EPAGRI. Normas técnicas para cultivo de capim-limão, citronela, palma-rosa e patchuli. Florianópolis, 2004. 58 p. (Epagri. Sistemas de Produção, 37).

8. FOCHESATO, M.L.; MARTINS, F.T.; SOUZA, P.V.D.; SCHWARZ, S.F.; BARROS, I.B.I. Propagação de louro (Laurus nobilis L.) por estacas semilenhosas com diferentes quantidades de folhas e tratadas com ácido indolbutírico. Revista Brasileira de Plantas Medicinais, v. 8, n. 3, p. 72-77. 2006.

9. GUENTHER, E. The essential oils: individual essential oils of the plant families Rutaceae and Labiatae. Malabar: Krieger. 1972. v. 3. 777 p.

10. HARTMANN, H.T.; KESTER, D.E.; DAVIES JR., F.T.; GENEVE, R.L. Plant propagation: principles and practices. 7. ed. New Jersey: Prentice Hall, 2002. 880 p.

11. LIMA, N.P.; BIASI, L.A. ; ZANETTE, F.; NAKASHIMA, T. Estaquia semilenhosa e análise de metabólitos secundários de guaco (Mikania glomerata Sprengel e Mikania laevigata Schultz Bip. Ex Baker). Revista Brasileira de Plantas Medicinais, v. 5, n. 2, p. 47-54, 2003.

12. MINAMI, K. Produção de mudas de alta qualidade em horticultura. São Paulo: T.A. Queiroz, 1995. 128 p.

13. NACHTIGAL, J.C.; HOFFMANN, A.; KLUGE, R.A.; FACHINELLO, J.C.; MAZZINI, A.R. de A. Enraizamento de estacas semilenhosas de araçazeiro (Psidium cattleyanum Sabine) com o uso do ácido indolbutírico. Revista Brasileira de Fruticultura, v. 16, n. 1, p. 229-235, 1994.

14. NEGRELLE, R.R.B.; DONI, M.E. Efeito da maturidade dos ramos na formação de mudas de guaco por meio de estaquia. Horticultura Brasileira, v.19, n. 3, p. 351-355. 2001.

15. NICOLOSO, F.T.; FORTUNATO, R.P.; FOGACA, M.A. de F. Influência da posição da estaca no ramo sobre o enraizamento de Pfaffia glomerata (Spreng.) Pedersen em dois substratos. Ciência Rural, v. 29, n. 2, p. 277-283. 1999.

16. PEREIRA, F.M.; OIOLI, A.A.P.; BANZATTO, D.A. Enraizamento de diferentes tipos de estacas enfolhadas de goiabeira (Psidium guayava L.) em câmaras de nebulização. Cientifica, v. 11, n. 2, p. 239-244, 1983.

17. REUVENI, O.; RAVIV, M. Importance of leaf retention to rooting of avocado cuttings. Journal of the American Society for Horticultural Science, v. 106, n. 2, p.127-130, 1981.

18. TAIZ, L.; ZEIGER, E. Fisiologia vegetal. 3 ed. Porto Alegre: Artmed, 2004. 719 p.

Recebido em 07/03/2007

Aceito em 31/08/2007 